\title{
The Diagnosis and Treatment of Adult Patients with SAPHO Syndrome: Controversies Revealed in a Multidisciplinary International Survey of Physicians
}

\author{
Victoria Furer (D) - Mitsumasa Kishimoto - Shigeyoshi Tsuji • \\ Yoshinori Taniguchi · Yoko Ishihara · Tetsuya Tomita • \\ Philip S. Helliwell · Ori Elkayam
}

Received: August 12, 2020 / Accepted: September 10, 2020 / Published online: September 24, 2020

(C) The Author(s) 2020

\section{ABSTRACT}

Introduction: This study aimed to investigate the current practice in the diagnosis and treatment of SAPHO syndrome among the

Digital Features To view digital features for this article go to https://doi.org/10.6084/m9.figshare.12933134.

V. Furer $(\bowtie) \cdot$ O. Elkayam

Department of Rheumatology, Tel Aviv Sourasky

Medical Center, Sackler Faculty of Medicine, Tel

Aviv University, Tel Aviv, Israel

e-mail: furer.rheum@gmail.com

M. Kishimoto

Department of Nephrology and Rheumatology,

Kyorin University School of Medicine, Tokyo, Japan

S. Tsuji

Department of Rheumatology and Orthopaedic Surgery, Osaka Minami Medical Center, Osaka, Japan

\section{Y. Taniguchi}

Department of Endocrinology, Metabolism,

Nephrology and Rheumatology, Kochi Medical

School Hospital, Kochi University, Nankoku, Japan

Y. Ishihara

Japan Medical Research Foundation, Tokyo, Japan

T. Tomita

Department of Orthopaedic Biomaterial Science, Osaka University Graduate School of Medicine,

Osaka, Japan

P. S. Helliwell

Leeds Institute of Molecular and Musculoskeletal

Medicine, University of Leeds, Leeds, UK international rheumatology and dermatology communities.

Methods: We conducted an electronic survey among the members of the Group for Research and Assessment of Psoriasis and Psoriatic Arthritis (GRAPPA), the Japan Spondyloarthritis, and Israeli Societies of Rheumatology.

Results: A total of 78 physicians participated in the survey: rheumatologists $(83 \%, n=65)$, dermatologists $(11.5 \%, n=9)$, and orthopedics $(3.8 \%, n=3)$. SAPHO was considered a subtype of spondyloarthritis by $48.7 \%(n=38)$, a subtype of psoriatic arthritis by $19.2 \%(n=15)$, a separate entity by $25.6 \%(n=20)$, and a subtype of reactive arthritis by $6.4 \% \quad(n=5)$. Palmoplantar pustulosis was the most prevalent cutaneous manifestation $(n=44,56.4 \%)$ and anterior chest pain-the most prevalent osteoarticular manifestation $(n=66,84.6 \%)$. The majority $(84.6 \%, n=66)$ voted for the update of the present diagnostic criteria by Khan 1994. Magnetic resonance imaging was considered the preferred imaging modality for the diagnosis of SAPHO by $41 \%(n=32)$. Conduction of bone biopsy for diagnosis of noninfectious osteitis was supported only by $10.3 \%$ $(n=8)$. Patient-reported outcomes were considered the most appropriate measure for the assessment of disease activity by $47.4 \%(n=37)$. The treatment approach was overall similar among the rheumatology and dermatology communities, including non-steroidal anti-inflammatory drugs, bisphosphonates, 
conventional disease-modifying anti-inflammatory drugs, and biologics.

Conclusions: Our study underlines the controversy on diagnosis and treatment of SAPHO syndrome among specialists in rheumatology and dermatology and emphasizes an unmet need for update and validation of diagnostic criteria and treatment approach.

Keywords: Diagnosis; Psoriatic arthritis; SAPHO; Spondyloarthritis; Survey SAPHO syndrome; Treatment

\section{Key Summary Points}

Controversy on diagnosis and treatment of synovitis, acne, pustulosis, hyperostosis, and osteitis (SAPHO) syndrome is reflected in an international survey.

There is an unmet need for update and validation of diagnostic criteria and treatment approach to SAPHO.

Multidisciplinary international collaboration is warranted to expand studies of SAPHO syndrome.

\section{DIGITAL FEATURES}

This article is published with digital features to facilitate understanding of the article. To view digital features for this article go to https://doi. org/10.6084/m9.figshare.12933134.

\section{INTRODUCTION}

The SAPHO syndrome (acronym for synovitis, acne, pustulosis, hyperostosis, and osteitis), introduced by Chamot in 1987, represents a rare heterogeneous disease mainly targeting the skin and the skeleton [1]. The true incidence and prevalence of SAPHO are unknown, as the syndrome is commonly under-recognized or misdiagnosed. SAPHO mainly affects children and young adults, often diagnosed as chronic recurrent multifocal osteomyelitis (CRMO) in the young population. Clinical manifestations of SAPHO are variable and share common features with psoriatic arthritis (PsA) and spondyloarthritis (SpA)-related diseases. The main cutaneous manifestations include palmoplantar psoriasis, severe acne, and less commonly, hidradenitis suppurativa. Musculoskeletal manifestations typically include a non-infectious osteitis, hyperostosis, and synovitis of the anterior chest wall, with the sternoclavicular junction being commonly affected, followed by the spine, sacroiliac joints, and peripheral nonerosive arthritis. Skin lesions may precede, follow, or occur simultaneously with the onset of musculoskeletal manifestations [2, 3]. Several clinical patterns of SAPHO have been recognized, including a relapsing-remitting or chronic disease in the majority of cases and rarely a monophasic disease [4]. Non-infectious sterile osteitis with subsequent hyperostosis resulting in osteolytic and osteosclerotic bone lesions represent a distinct pathological and radiographic feature of SAPHO [5]. Etiopathogenesis of SAPHO is considered multifactorial. Low virulent pathogens, such as Propionibacterium acnes, may trigger an exaggerated inflammatory response of the bone marrow in genetically susceptible individuals, leading to a form of "reactive osteitis" [6].

Diagnostic criteria for SAPHO remain preliminary and lack validation. The first diagnostic criteria proposed by Chamot were based on the clinical grounds, including a wide spectrum of clinical features [1], further followed by criteria proposed by Kahn requiring pathological evidence of osteitis or osteomyelitis for establishing the diagnosis, with or without typical skin lesions [7]. The modified criteria proposed by Hayem, based on an observational cohort of 120 patients with SAPHO, suggested to base the diagnosis on the combination of typical osteoarticular and skin manifestations, following the exclusion of inflammatory bowel disease, bone infection, and tumors [8]. To date, no formal guidelines outlining a diagnostic approach to SAPHO exist. Bone scintigraphy is commonly used as first-line imaging in the systemic evaluation of osteoarticular lesions in 
SAPHO syndrome [9]. In view of advances in imaging techniques, magnetic resonance imaging (MRI), computerized tomography (CT), and ultrasonography (US) application of an appropriate imaging modality for diagnosis and monitoring of disease activity is highly needed in patients with SAPHO, along with avoidance of unnecessary imaging tests. No specific core domain set of outcome measures were developed or applied in SAPHO. Furthermore, there are no evidence-based treatment algorithms in SAPHO due to a lack of clinical trials in this rare medical condition. Treatment choice is based on retrospective reports and case series. In fact, a wide spectrum of medications has been used to treat SAPHO, extrapolated from treatment approaches to psoriasis, severe acne, PsA, and $\mathrm{SpA}$. To date, there are no data on long-term efficacy, adverse events, and outcomes of different treatments in SAPHO [10].

SAPHO is a rare disease involving skin and rheumatologic manifestations, with a potentially complicated and severe course. Clinical presentation may pertain to several specialties, including rheumatology, dermatology, orthopedics, physiotherapists, and pediatricians. Optimal management of these patients requires multidisciplinary collaborative care. The Group for Research and Assessment of Psoriasis and Psoriatic Arthritis (GRAPPA) is an international network platform of medical disciplines treating psoriasis and psoriatic arthritis and recent interest in this condition was reflected in a symposium on pustular psoriasis and SAPHO during the recent GRAPPA virtual annual meeting in July 2020 (www.grappanetwork.org). To further explore current concepts and practice on the approach and treatment strategies in SAPHO, a survey was conducted among the members of GRAPPA as well as the Japanese Sponsyloarthritis and Israeli Societies of Rheumatology, and the results are presented here.

\section{METHODS}

An electronic online survey was conducted among the members of GRAPPA, Japanese Spondyloarthritis, and Israeli Societies of Rheumatology. The questionnaire was distributed to 703 physicians, including 26 members of the Japanese Spondyloarthritis Society, 70 members of Israeli Society of Rheumatology, and 613 GRAPPA members (one Japanese responder and five Israeli responders belonged to GRAPPA as well). No selection criteria were applied. The survey took place in 2017, using a Google Forms platform. The language of the survey was English. The study, considered as less than 'minimal risk research', was certified as exempt by the Tel Aviv Sourasky Medical Center Institutional Review Board (Helsinki Committee) and participants' written informed consent was waived as the participation in the study was voluntarily and each participant had an option to fill out the survey anonymously. In the survey, responders were asked to state their medical subspecialty and the country of origin. SAPHOrelated questions included the total number of SAPHO patients under a physician's care, the annual number of new SAPHO cases, the most commonly encountered osteoarticular and skin manifestations, and preferable imaging modality for the diagnosis of SAPHO in the clinical practice. The use of ultrasound in the assessment of sternoclavicular or other sternal joints and the performance of bone biopsy in cases of osteitis or hyperostosis for the diagnosis of SAPHO was posed. The responders were asked whether the 1994 diagnostic criteria for SAPHO reflected the characteristics of SAPHO patients in their practice and whether there was a need for the update or modification of the SAPHO diagnostic criteria. Responders' points of view on the classification of the SAPHO syndrome to one of the following diseases' subgroups: psoriatic arthritis, spondyloarthropathy, reactive arthritis, or a separate independent entity were evaluated. Further, measures for the assessment of disease activity were inquired. Survey participants were questioned about the treatment approach to SAPHO and whether there was a difference in the treatment approach in patients with psoriasis versus acne.

\section{RESULTS}

A total of 78 physicians completed the survey, reflecting a response rate of $11.1 \%$. Among the 


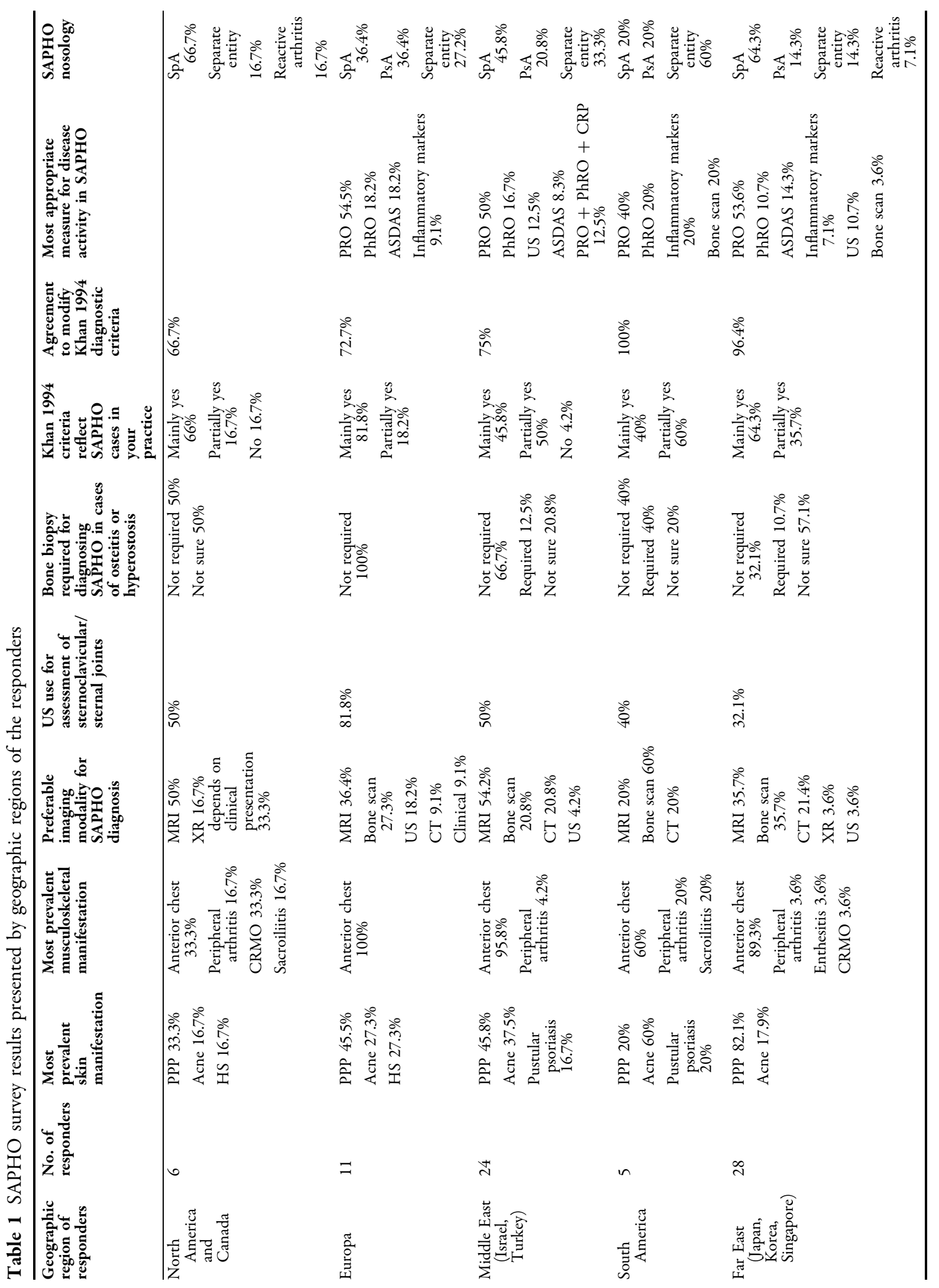




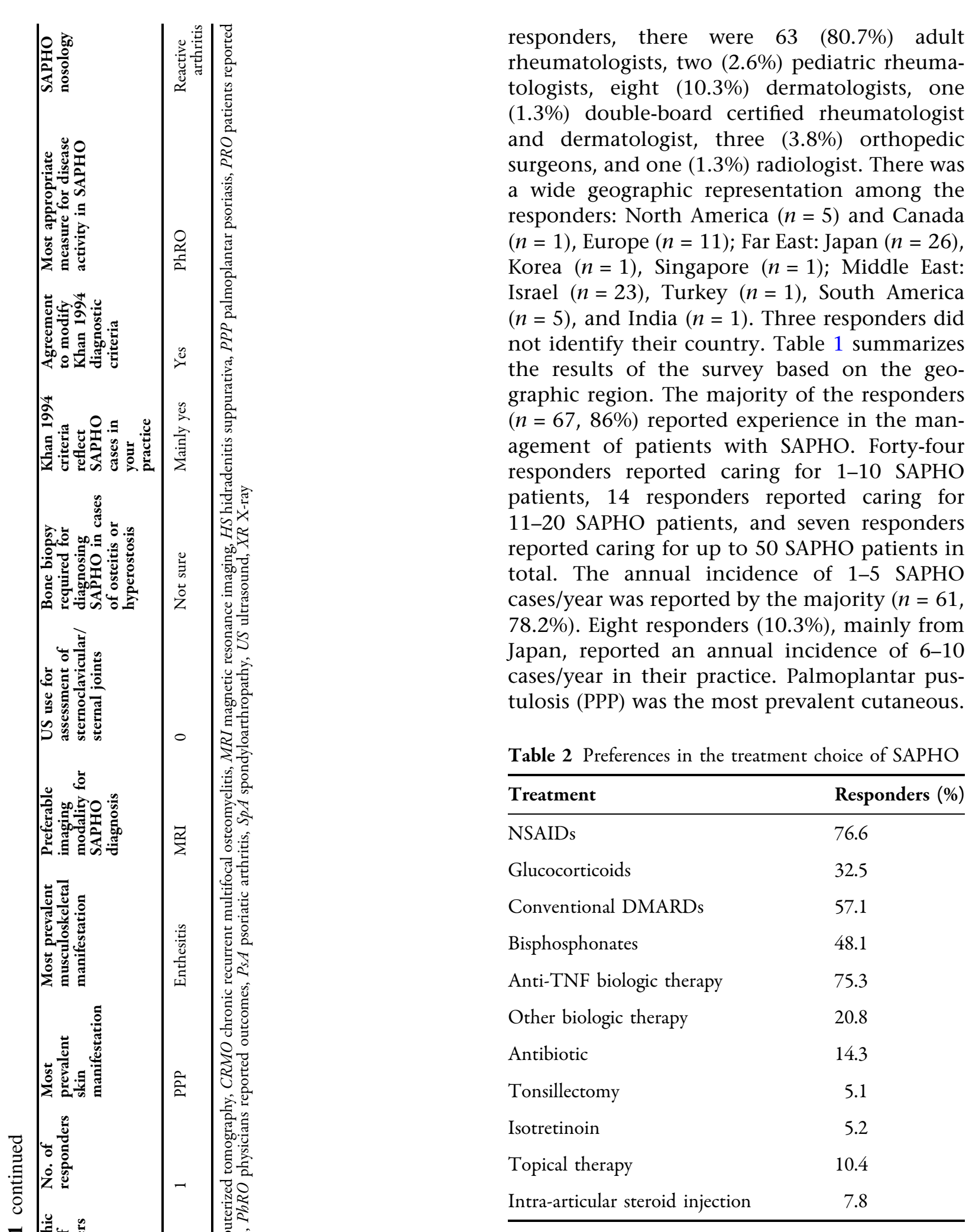

NSAIDs non-steroidal anti-inflammatory drugs, DMARDs disease-modifying anti-rheumatic drugs 
Manifestation among all regions $(n=44$, $56.4 \%)$, with a particularly high prevalence in Japan $(n=23,82.1 \%)$, followed by acne $(n=20$, $25.6 \%)$, pustular psoriasis $(n=7,9 \%)$, and rarely hidradenitis suppurativa $(n=5,5.1 \%)$. Anterior chest pain and swelling (osteitis, hyperostosis, and joint inflammation of the anterior chest bones and joints) was the most prevalent osteoarticular manifestation $(n=66$, $84.6 \%)$, followed by peripheral arthritis $(n=5$, $6.4 \%)$, sacroiliitis $(n=2,2.6 \%)$, and enthesitis $(n=2,2.6 \%)$. Chronic recurrent multifocal osteomyelitis (CRMO) was reported by three responders (3.8\%). MRI was considered the preferred imaging modality for SAPHO diagnosis by $41 \%(n=32)$ of the responders, whereas $26.9 \%(n=21)$ preferred bone scan, and $19.2 \%$ $(n=15)$ voted for computerized tomography (CT) scan. Four responders (5.1\%) reported using US applied for sternum joints for SAPHO diagnosis. Regarding the indication for bone biopsy to confirm the diagnosis of SAPHO in cases of osteitis or hyperostosis, only $10.3 \%$ $(n=8)$ supported the conduction of biopsy, whereas 55\% $(n=43)$ found no need for this test in the diagnostic work-up of SAPHO. A third of the responders $(34.6 \%)$ were uncertain regarding this item. Whereas $59 \%(n=46)$ stated that the Khan diagnostic criteria mainly reflected SAPHO cases in their practice, the vast majority $(84.6 \%, n=66)$ still voted for modification and update of these criteria. SAPHO was considered as a subtype of SpA by $48.7 \%$ $(n=38)$, a subtype of PsA by $19.2 \%(n=15)$, a separate entity by $25.6 \%(n=20)$, and reactive arthritis subtype by $6.4 \%(n=5)$. Patient-reported outcomes, including patient global and pain assessment (VAS), were considered the most appropriate measures for assessment of disease activity by $47.4 \%(n=37)$, followed by clinical physician disease assessment by $16.7 \%$ $(n=13)$, Ankylosing Spondylitis Disease Activity Score (ASDAS) by $10.3 \%(n=8)$, and blood inflammatory markers by $9 \%(n=7)$. Six $(7.7 \%)$ responders suggested a follow-up of disease activity by US, $2.6 \%(n=2)$ by bone scan, and one $(1.3 \%)$ by MRI. Three responders $(3.8 \%)$ suggested a combination of clinical, laboratory, and imaging measures for assessment of disease activity and follow-up. The list of preferable medications and treatments used for SAPHO is presented in Table 2 . The question related to the treatment approach to SAPHO was formulated as a multiple-choice question, presenting a list of potential therapeutics. Overall, the treatment approach was similar among the rheumatology and dermatology responders. Non-steroidal anti-inflammatory drugs (NSAIDs) were the first choice universally listed by most responders (colchicine was not specifically mentioned), followed in decreasing order of frequency by anti-TNFa biologics, conventional DMARDs, bisphosphonates, other biologics (not specifically named), and finally antibiotics. Remarkably, only Japanese responders $(n=4)$ suggested tonsillectomy as an additional mode of treatment. Comparing treatment approaches among different regions, European and Middle East responders reported a significantly more common use of bisphosphonates $(63.6 \%, n=7$ and $62.5 \%, n=15$, respectively) compared to Japanese responders $(n=11,39.3 \%)$. Whereas $27.3 \%(n=3)$ of European and 17.8\% $(n=5)$ of Japanese responders reported the use of antibiotics, none of the Israeli responders used this treatment.

\section{DISCUSSION}

Our survey provides insight into the current approach and treatment practices of an international community of physicians, mainly rheumatologists and dermatologists, experienced in the care of patients with SAPHO. This syndrome is not only rare and heterogeneous but also presents in different subsets around the globe. SAPHO manifestations in Asian and Caucasian populations show different characteristics. Asian patients with SAPHO mainly present with PPP and only rarely with severe acne or hidradenitis suppurativa. In fact, pustulotic arthro-osteitis (PAO) is the most common form of SAPHO in the Japanese population. PAO was first reported by Sonozaki et al. in 1979 based on a case series of 22 cases with PPP and anterior chest involvement [11], further expanded to 53 cases [12]. Histologic examination of the anterior wall tissues revealed non-suppurative chronic 
inflammation of the soft tissues around the sterno-costo-clavicular region or nonspecific chronic inflammation of bones. Spondylitis, sacroiliitis, and peripheral non-erosive arthritis were observed in up to $30 \%$ of this cohort. All patients were HLA-B27 negative. Whether PAO in Japan is a genetically and clinically distinct entity or not requires further investigation. Radiologically, spinal lesions are more common in Asians versus Caucasians [4, 13-15]. Limited reports point to a particularly severe form of SAPHO in the African-American population, characterized by acne fulminans and hidradenitis suppurativa [16]. There is a lack of data on SAPHO characteristics from other geographic regions. Our survey seems to be unique representing an insight into the features of SAPHO among a widespread community of rheumatologists and dermatologists. In terms of clinical features, PPP represented the most common cutaneous manifestation across the globe, followed by acne in the Middle East and European communities. Hidradenitis suppurativa was reported only in the North American, Canadian, and European communities. The involvement of the anterior chest wall was the most common osteoarticular manifestation, consistent with the observational data from Europe [15] and Japan [14]. The prevalence of peripheral arthritis, enthesitis, sacroiliitis, and CRMO was variable in different geographic regions. The nosology of SAPHO was mainly viewed as a subtype of SpA, followed by PsA subtype, except for the South American subgroup, which categorized SAPHO as a separate and independent entity. The survey demonstrated a wide range of opinions regarding the preferred imaging modality in SAPHO, ranging from MRI (most commonly applied in North America, Canada, and the Middle East), CT (equally applied in the Far East, South America, and the Middle East), USA (most commonly applied in Europe), and bone scan (most commonly applied in South America). Notably, the choice of the imaging modality should be dependent on the stage of the disease, as early and late radiographic findings significantly differ. For example, early lesions tend to be osteodestructive and best demonstrated by MRI or bone scintigraphy, whereas late lesions tend to be osteoproliferative, resulting in hyperostosis and sclerosis that are best assessed on CT scanning [9]. Further, a discussion regarding the need for bone biopsy in the diagnosis of SAPHO-related osteitis was demonstrated, with the majority of responders voting for no need for biopsy (for example, 100\% of European responders) or being unsure regarding this item. Consensus for the update of the diagnostic criteria was reached across all regions. Other discussion points included the measures for disease assessment and the therapeutic approach.

Limitations of our study relate to the inherent limitations of the study design as a survey. Notably, there was a low response rate, which might be explained by the rarity of SAPHO syndrome around the globe. In fact, some of the responders reported no personal experience with SAPHO patients. Yet, we decided to include their views on the diagnosis and treatment of SAPHO, as they reflected the current concepts on SAPHO of the corresponding medical society. A discrepancy in recall accuracy of the responders was another inherent limitation of the survey design. Furthermore, the questionnaire used in this survey was in particular constructed for this study, without prior validation. All the mentioned limitations might potentially affect the validity of the study.

\section{CONCLUSIONS}

In summary, the results of our survey reflect the diversity in the current diagnostic and therapeutic approach to SAPHO syndrome in clinical practice. An unmet need for the update, modification, and validation of the SAPHO diagnostic criteria and setting disease activity measures was reflected by most of the participants. Future directions include establishing an international registry for SAPHO, which will lead to the development of classification and diagnostic criteria for SAPHO.

\section{ACKNOWLEDGEMENTS}

We thank all the survey participants. 
Funding. No funding or sponsorship was received for this study or publication of this article.

Authorship. All named authors meet the International Committee of Medical Journal Editors (ICMJE) criteria for authorship for this article, take responsibility for the integrity of the work as a whole, and have given their approval for this version to be published.

Disclosures. Victoria Furer, Mitsumasa Kishimoto, Shigeyoshi Tsuji, Yoshinori Taniguchi, Yoko Ishihara, Tetsuya Tomita, and Ori Elkayam have nothing to disclose. Philip S. Helliwell is a member of the journal's Editorial Board.

Compliance with Ethics Guidelines. The study, considered as less than 'minimal risk research', was certified as exempt by the Tel Aviv Sourasky Medical Center Institutional Review Board (Helsinki Committee) and participants' written informed consent was waived as the participation in the study was on a voluntarily and each participant had an option to fill out the survey anonymously.

Data Availability. The datasets generated during and/or analyzed during the current study are available from the corresponding author on reasonable request.

Open Access. This article is licensed under a Creative Commons Attribution-NonCommercial 4.0 International License, which permits any non-commercial use, sharing, adaptation, distribution and reproduction in any medium or format, as long as you give appropriate credit to the original author(s) and the source, provide a link to the Creative Commons licence, and indicate if changes were made. The images or other third party material in this article are included in the article's Creative Commons licence, unless indicated otherwise in a credit line to the material. If material is not included in the article's Creative Commons licence and your intended use is not permitted by statutory regulation or exceeds the permitted use, you will need to obtain permission directly from the copyright holder. To view a copy of this licence, visit http://creativecommons.org/licenses/by$\mathrm{nc} / 4.0 /$.

\section{REFERENCES}

1. Chamot AM, Benhamou CL, Kahn MF, Beraneck L, Kaplan G, Prost A. Acne-pustulosis-hyperostosisosteitis syndrome. Results of a national survey 85 cases. Rev Rhum Mal Osteoartic. 1987;54(3): 187-96.

2. Rukavina I. SAPHO syndrome: a review. J Child Orthop. 2015;9(1):19-27.

3. Firinu D, Garcia-Larsen V, Manconi PE, Del Giacco SR. SAPHO syndrome: current developments and approaches to clinical treatment. Curr Rheumatol Rep. 2016;18(6):35.

4. Colina M, Govoni M, Orzincolo C, Trotta F. Clinical and radiologic evolution of synovitis, acne, pustulosis, hyperostosis, and osteitis syndrome: a singlecenter study of a cohort of 71 subjects. Arthritis Rheum. 2009;61(6):813-21.

5. Jurik AG, Klicman RF, Simoni P, Robinson P, Teh J. SAPHO and CRMO: the value of imaging. Semin Musculoskelet Radiol. 2018;22(2):207-24.

6. Assmann G, Simon P. The SAPHO syndrome-are microbes involved? Best Pract Res Clin Rheumatol. 2011;25(3):423-34.

7. Kahn MF, Khan MA. The SAPHO syndrome. Baillieres Clin Rheumatol. 1994;8(2):333-62.

8. Hayem G. SAPHO syndrome. Rev Prat. 2004;54(15): $1635-6$.

9. Schaub S, Sirkis HM, Kay J. Imaging for synovitis, acne, pustulosis, hyperostosis, and osteitis (SAPHO) syndrome. Rheum Dis Clin North Am. 2016;42(4): 695-710.

10. Carneiro S, Sampaio-Barros PD. SAPHO syndrome. Rheum Dis Clin North Am. 2013;39(2):401-18.

11. Sonozaki H, Azuma A, Okai K, Nakamura K, Fukuoka S, Tateishi A, et al. Clinical features of 22 cases with "inter-sterno-costo-clavicular ossification". A new rheumatic syndrome. Arch Orthop Trauma Surg. 1979;95(1-2):13-22.

12. Sonozaki H, Mitsui H, Miyanaga Y, Okitsu K, Igarashi M, Hayashi Y, et al. Clinical features of 53 cases with pustulotic arthro-osteitis. Ann Rheum Dis. 1981;40(6):547-53. 
13. Li C, Zuo Y, Wu N, Li L, Li F, Zhang W, et al. Synovitis, acne, pustulosis, hyperostosis and osteitis syndrome: a single-centre study of a cohort of 164 patients. Rheumatology (Oxford). 2016;55(6): 1023-30.

14. Okuno H, Watanuki M, Kuwahara Y, Sekiguchi A, Mori Y, Hitachi S, et al. Clinical features and radiological findings of 67 patients with SAPHO syndrome. Mod Rheumatol. 2018;28(4):703-8.
15. Salles M, Olive A, Perez-Andres R, Holgado S, Mateo L, Riera E, et al. The SAPHO syndrome: a clinical and imaging study. Clin Rheumatol. 2011;30(2): 245-9.

16. Steinhoff JP, Cilursu A, Falasca GF, Guzman L, Reginato AJ. A study of musculoskeletal manifestations in 12 patients with SAPHO syndrome. J Clin Rheumatol. 2002;8(1):13-22. 